Clinical Risk Index for Babies score II (CRIB II score) is a five-items scoring system to predict initial risk of mortality among neonates less than 32 weeks of gestation. Thus, making it an easy, quick and economical tool for early detection of preterm neonates' mortality risk in resource limited busy centres in India.

Objectives Objective:

To validate the efficacy of Clinical Risk Index for Babies score II in predicting pre-discharge neonatal mortality in early preterm neonates needing intensive care in a high resource tertiary care centre providing referral services in central India.

Methods Methods:

In this prospective observational study, after taking informed consent of parents and ethical committee clearance, the CRIB-II scores, which include birthweight, gestational age, sex, body temperature, and base excess, were recorded within the first hour of admission for 140 neonates of gestational age ranging between 28-31 weeks and birth weight ranging from $1000 \mathrm{~g}$ to $2499 \mathrm{~g}$; of both sexes born in a tertiary care institute of central India and admitted to its neonatal intensive care unit (NICU). Babies of gestation $<28$ weeks and $>31$ completed weeks, birth weight $<1000$ g, having lethal congenital malformations, delivery room deaths and those admitted after $1 \mathrm{hr}$ of birth were excluded. Outcome measure was in hospital death or discharge. The sensitivity and specificity of CRIB-II scores and its cut off point to predict mortality were examined using Receiver Operating Characteristic curves (ROC) with area under curve (AUC) indicating predictive accuracy. Its association with mortality was determined by Cox Regression Hazard analysis. Clinical parameters were compared between Non-survivors and survivors by performing independent t-test. A $\mathrm{p}<0.05$ was considered as statistically significant.

Results Results:

Male: female ratio was 0.92:1. The mean Gestational age was $30.27 \pm 0.89$ weeks, mean birth weight being 1599.75 \pm 282.35 g. CRIB II score ranged from 1-19 with a mean of $13.16 \pm 25.56$ among non survivors and mean of $5.66 \pm$ 2.24 among the survivors ( $\mathrm{p}$ value $=<0.0001$ ). The total mortality in the study was $47.1 \%(66 / 140)$. There was a progressive increase in mortality with increasing CRIB II score $(\mathrm{p}=0.001)$ and increase in survival with increasing birth weight, gestational age, body temperature and hospital stay ( $\mathrm{p}$ $<0.0001$ for each variable). CRIB II score $\geq 9$ cut off was found to be significantly associated with neonatal mortality with sensitivity, predictive value and specificity of $95.65 \%$, $95.65 \%$ and $95.77 \%$ respectively $(\mathrm{p}<0.001 ; 95 \%$ CI 0.98 (0.96-1.00); hazard ratio $=1.38)$. The ROC curve for CRIB II score was suggestive of AUC of 0.9868 ie $98 \%$ predictive accuracy.

Conclusions Conclusion:

The present study shows that the CRIB II score is a useful and reliable tool to prioritize the interventions in NICUs and will help to reduce the neonatal mortality rate and improve preterm neonates' survival in India if used appropriately in tertiary care centres.

\section{NEONATAL ECTOPIC ATRIAL TACHYCARDIA- MIND THE GAP (PR INTERVAL)!}

Ahmed Kamal, Pramod Nair. UK

10.1136/bmjpo-2021-RCPCH. 141
Background Whereas SVTs are well recognised in neonates with early diagnosis and management occasionally ectopic atrial tachycardia in neonates do happen infrequently and could be mis diagnosed as sinus tachycardia particularly if in the slower range

Objectives The tachycardia in these instances often tend to be incessant and can lead to tachycardia induced cardiomyopathy if the rate remains uncontrolled. The ECG changes are subtle and needs a trained eye to pick up this diagnosis.

Methods We present a case of a male infant who presented acutely to the hospital at age of 19 days with blood in the stool. He was born at term baby with mild intra uterine growth retardation but otherwise well at birth. He was noted to have hypospadias and was diagnosed as a case of cow's milk protein intolerance. During the admission he was incidentally noted to be tachycardic with a heart rate consistently between 180 and 200 beats per minute while otherwise being well and afebrile. His ECG showed narrow complex tachycardia at a rate between 180 to 200 beats per minute. Blood tests were overall satisfactory with an elevated troponin level and negative viral serology studies. An echocardiography done suggested mild left atrial and left ventricular dilatation with mild impairment of function. The case was discussed with the tertiary Cardiac team who felt this was more likely to be a sinus tachycardia and the patient was subsequently discharged. At age of 6 weeks, the child was seen in the outreach cardiac clinic. The ECGs (Current and previous were reviewed). It was noted that $\mathrm{P}$ waves were normal axis but negative in aVL, the PR interval was disproportionately long for the tachycardia. This suggested atrial tachycardia which was persistent. An echocardiogram showed a structurally normal heart with mild left heart volume overload and mildly impaired left ventricular function. The child was stuck in a slow atrial tachycardia rhythm which contributed towards the impaired function. Propranolol was started at a dose of $1 \mathrm{mg} / \mathrm{kg}$ TDS.

Results At further follow up at 7 months, the child was thriving and well, echocardiography showed that the cardiac dimensions were normal and the function had improved. ECGs and ambulatory monitoring showed normal rates and rhythms. The beta blockers were continued with a plan to wean in future.

Conclusions Ectopic atrial tachycardia (EAT) in a neonate can present with incessant tachycardia but can be in a slower range of 180-200 bpms. The ECG changes can be subtle with normal $\mathrm{p}$ waves ( $\mathrm{P}$ wave axis could be abnormal) but the persistent tachycardia, disproportionately long PR interval for the tachycardia and impairment of ventricular function could be useful pointers towards this diagnosis. EAT could lead to impairment of ventricular function if poorly controlled. Medications to control the tachycardia can help with eventual recovery of ventricular function as this case has highlighted.

\section{NEONATAL READMISSIONS TO THE PAEDIATRIC WARDS DURING THE FIRST WAVE OF THE PANDEMIC: 3 CENTRE STUDY IN NORTHERN IRELAND}

Katarina Stefkova, Andrea Stobo, Laura Johnston, David Grier, Chloe Lynas. UK

\subsection{6/bmjpo-2021-RCPCH.142}

Background Readmissions of infants younger than 28 days back into hospital are highly undesirable. It is upsetting for the families and puts babies at risk of hospital acquired 
infection. We have subjectively noted an increase in readmissions, so decided to research the number of readmissions and reflect on the factors associated with these

Objectives To study the number of readmissions in infants under 28 days into the paediatric wards, and to see whether there has been an increase compared to 2019. To compare the data between the hospitals in our region.

Methods The project was done as a retrospective study in 3 separate hospitals in Northern Ireland- Antrim Area Hospital, Craigavon Area Hospital and the Royal Belfast Hospital for Sick Children (RBHSC). We looked at the neonatal readmissions in infants under 28 days of age, we excluded infants needing intensive care after birth.

The timeline we studied was between 1st April and 1st July in 2020, which was the height of the first wave of the Coronavirus pandemic in Northern Ireland. We looked at the main reason for readmission in each infant, comparing the data with the readmission rates and causes in 2019.

We also looked at the NIMATS documents of the infants who were readmitted to establish their length of stay in the postnatal ward before discharge.

Results Overall, the number of neonatal readmissions varied among the three hospitals studied.

In Craigavon Area Hospital, the total number of readmissions was 50 in 2020, and 40 in 2019. This signifies a 20\% increase in neonatal readmissions.

In Antrim Area Hospital, the total number of neonatal readmissions was 46 in 2020, compared to 62 in 2019.

The RBHSC, the tertiary centre, also noted a decrease of neonatal readmissions, from 37 in 2019 to 25 in 2020.

In regard to the length of postnatal ward stay, in all of the hospitals studied, it was reduced in 2020. In Antrim hospital, the mean length of stay in postnatal ward in the infants needing readmitted went from 44.3 hours in 2020 , to 37.5 hours in 2019. In Craigavon, the mean length of stay in postnatal ward was $44.7 \mathrm{hrs}$ in 2019, compared to $38.5 \mathrm{hrs}$ in 2020. In the RBHSC, the mean length of stay was 45.2 in 2019 and reduced to $29.1 \mathrm{hrs}$ in 2020 .

The leading cause of readmissions was jaundice in both Craigavon Area hospital and Antrim Area hospital in 2020 and 2019.

Conclusions It was interesting to see such variation in neonatal readmissions among three hospitals in the same country.

There has been an overall increase of readmissions for infants with poor feeding, which could be explained by less support available for mums during the pandemic. This could have been due to overall shorter stay in the postnatal ward, dads not being allowed into the postnatal ward, and less frequent midwife visits.

A decrease of conditions such as bronchiolitis could be explained by less mixing of newborn infants with older children.

\section{THE IMPACT OF COVID-19 ON THE MENTAL HEALTH OF YOUNG PEOPLE AGED 16-19 AND THEIR ATTITUDES TOWARDS VACCINATION}

Sahana Balamurugan, Balamurugan Thyagarajan. UK

10.1136/bmjpo-2021-RCPCH.143
Background Little is known about the impact of COVID-19 on the mental health of young people aged 16-19 or their attitudes towards COVID-19 vaccination. Social media suggests this demographic is not coping positively with the new changes implemented by the pandemic. Collecting data on their opinions may help with planning support for this generation of young people.

Objectives To explore the mental health impacts of the Coronavirus pandemic and the attitude toward coronavirus vaccines on young people aged 16-19, living in the UK.

Methods Links were posted on Instagram and Snapchat inviting students aged 16-19 to partake in an anonymous online survey during the last week of December 2020. The survey reached 115 people; they were asked questions on the changes to their mental health, the effect of COVID19 and their attitude towards the newly developed vaccines.

Results 42/115 people responded to the survey, 95\% of which were sixth form students aged $16(65.9 \%)$ to $17(26.8 \%)$, mostly White British (82.9\%) or Asian (12.2\%). The majority of responders are in Tier 4(53.7\%) followed by Tier 2 (41.5\%); 78\% lived with $>4$ people. $2 / 42$ (5.0\%) had been diagnosed with COVID-19 whilst 90.0\% have had a friend/ family member diagnosed.

$25 / 41(61.0 \%)$ of the responder's felt their mental health worsened over the pandemic; 10/41(24.39\%) had a previously existing diagnosed mental health condition. The prevailing symptoms experienced included feeling anxious (83.0\%), getting frustrated easily $(78.0 \%)$, a foreboding sense (78.0\%), unexplained worrying $(70.7 \%)$ and difficulty relaxing (65.9\%). Respondents were significantly concerned about the pandemic affecting their education (85.4\%) and career choices (70.7\%).

When asked about home life, $47.5 \%$ have trouble studying, $31.7 \%$ have trouble concentrating, $24.4 \%$ have trouble sleeping and $12.2 \%$ have trouble eating. 14/41 (34.1\%) respondents express negative behaviour toward their families, $30.8 \%$ felt more isolated from their family and 28.2\% argue more with their family. Contrastingly, 23.1\% were talking more and $28.2 \%$ felt closer to their family. The thought of transmitting coronavirus to a vulnerable person $(90.0 \%)$ or transmitting coronavirus to a family member or friend $(75.5 \%)$ majorly worried the majority of respondents.

When asked about the newly developed vaccine, $70.0 \%$ expressed confidence in its safety while $53.6 \%$ felt following the vaccination their mental health could improve. $25.0 \%$ stated they would not take the vaccine if given a chance.

Respondents reckon less pressure at school (48.8\%), additional mental health support $(46.3 \%)$, having someone to talk to $(46.3 \%)$, information on where to receive support $(43.9 \%)$ and after school clubs (43.9\%) would improve their mental health.

Conclusions Over the pandemic, the mental health of people aged 16-19 has worsened - they are experiencing a range of negative emotions and insomnia. Respondents perceive a more dysfunctional relationship with their family. Many believe the pandemic has affected their education and will affect their future career. To combat this decline, they might need more mental health support and less pressure. A large percentage of them may not voluntarily take the coronavirus vaccine; this highlights the need for education about the origin and production of the vaccine to gain confidence in its safety. 